\title{
Jouer à la poupée chez Rousseau, Michelet et Beauvoir
}

\author{
Laurence Mall
}

The description of female childhood often includes the mention of dolls, privileged instruments of gender role edification. By studying three texts that describe the specificity of the little girl (Rousseau's Emile, Michelet's La Femme, and De Beauvoir's The Second Sex), this article aims to show the numerous functions held by the doll: substitute, synecdoque, symbol and symptom. The doll also acts as a double screen, revealing the heavy ideology present in the discourse where she herself appears. These texts do not present little girls who play with dolls, but grown women, sad or tragic figures of their author's haunted spirits.

Latin Pupa: petite fille et poupée (Littré)

Quelle petite fille est-on quand on joue à la poupée chez Jean-Jacques Rousseau, chez Jules Michelet et chez Simone de Beauvoir? Ou plutôt, sans doute, quelle femme promet-on d'être alors? Voici ce que cette étude se donne pour tâche d'éclairer. $^{\mathrm{i}}$

Mais d'abord, un détour du côté de la fiction ou des écrits personnels. Lorsque dans ces derniers sont décrites les relations entre une fillette et sa poupée elles sont individualisées, nuancées selon les circonstances et la personnalité de la petite hérö̈ne. Que l'on songe à la Cosette d'Hugo ou à la jeune Aurore Dupin de l'Histoire de ma vie: l'expérience est de facto première, et les réflexions qui lui confèrent une signification plus générale lui restent liées. George Sand se remémore une de ses poupées et ce n'est qu'ensuite qu'elle s'interroge sur la qualité de la croyance de l'enfant: « Le sentiment que les petites filles éprouvent pour leur poupée est véritablement assez bizarre, et je l'ai ressenti si vivement et si longtemps que, sans l'expliquer, je puis aisément le définir. Il n'est aucun moment de leur enfance où elles se trompent entièrement sur le genre d'existence de cet être inerte [...] ", mais l'affection extrême qu'elles éprouvent est comparable à l'idolâtrie: à la dévotion des « sauvages » et des Anciens adorant leurs idoles (55354). C'est après avoir longuement évoqué la passion de Cosette pour la poupée de ses rêves que Hugo se lance dans une réflexion sur la maternité, au milieu de la scène où Jean Valjean vient chercher la fillette chez les Thénardier:

La poupée est un des plus impérieux besoins et en même temps un des plus charmants instincts de l'enfance féminine. Soigner, vêtir, parer, habiller, déshabiller, rhabiller, enseigner, un peu gronder, bercer, dorloter, endormir, se figurer que quelque chose est 
quelqu'un, tout l'avenir de la femme est là. Tout en rêvant et tout en jasant, tout en faisant de petits trousseaux et de petites layettes, [...] l'enfant devient jeune fille, la jeune fille devient grande fille, la grande fille devient femme. Le premier enfant continue la dernière poupée. $(521-22){ }^{\text {ii }}$

Ces généralités renforcent mais sans se substituer à elle l'analyse subtile ou dramatique du rapport entre la fillette et sa poupée dans une dimension existentielle très forte. C'est que ces écrivains savent " color[er] les joues de l'enfance » comme l'écrit Baudelaire à propos de Hugo (792). Les pages évoquées ci-dessus sont avant tout attachées à dévoiler un pan de l'expérience privée propre à l'enfant héroïne de ces deux textes: Cosette ou la jeune Aurore. Observation ou souvenir personnels, qu'importe ici: le jeu de la poupée est sérieux mais heureux, ouvrant sur un monde enchanté qui fait écrire à Sand, passant d'une description de sa poupée au Casse-noisette d'Hoffmann, que « l'imagination des enfants est aussi riche et aussi confuse que ces brillants rêves du conteur allemand » (556).

En contraste, je vais ici examiner des textes que l'on peut qualifier d'anthropologie sociale et culturelle: Émile ou de l'éducation (1762) de Rousseau, La Fermme (1859) de Michelet et Le Deuxième sexe (1949) de Beauvoir. Ces textes, séparés approximativement d'un siècle, sont en partie (Émile) ou entièrement consacrées aux femmes et comportent des pages sur les fillettes et le jeu de la poupée, étant entendu que, comme l'écrit Montaigne, " les jeux des enfants ne sont pas jeux, et les faut juger en eux comme leurs plus sérieuses actions » (108). Je me concentrerai ici sur les passages mettant en scène une ou la fillette et sa poupée pour déterminer ce qu'ils révèlent, économiquement mais dramatiquement, sur la conception de l'enfance féminine. Textes fameux, à forte personnalité, à forte écriture: que deviennent des idées parfois banales chez des auteurs qui ne le sont pas? Elles transgressent leur place assignée. On aura l'occasion de constater l'extraordinaire incapacité de ces trois auteurs à penser les petites filles comme êtres en soi; on pourra par conséquent repérer les phénomènes d'anticipation, d'amplification et de distorsion qui accompagnent leur traitement. La poupée est au coeur de ces phénomènes: miniature, elle provoque tous les grossissements; hors du temps, elle précipite sans relâche le discours sur la fillette dans le temps adulte; simulacre de l'être humain, en sens inverse elle appelle la comparaison de l'être humain avec elle: bébé ou coquette.

La pratique des poupées de mode (« Ce sont des figures d'enfans si proprement habillées et coëffées, qu'on les envoie dans les pays étrangers pour répan-

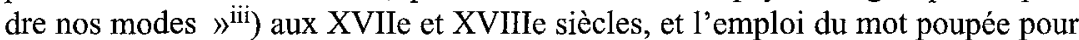
désigner les jolies femmes excessivement fardées et parées ne sont pas sans peser sur la conception du jeu et de la petite joueuse. L'existence du terme très proche de « poupon » pour « bébé » n'est pas non plus sans effet. Les brouillages temporels sont d'autant plus présents que le jeu de la poupée est d'abord vu comme une imitation des attitudes à venir et une initiation aux tâches futures. L'enfance contient l'âge adulte: le jeu de la poupée ne cesse que comme jeu. En effet chez nos auteurs on cesse de jouer à la poupée soit quand on devient poupée soi-même, 
soit quand on pouponne. On pourrait dire que la poupée lance un infernal système de synecdoque - infernal parce que réversible. Toutes les poupées sont-elles russes? La poupée est la petite fille en petit, qui est la femme en petit, qui ellemême se dévouant au plaire ou à la maternité se consacre à la miniature... Circularité incestueuse, tautologie, enfermement dans un cycle au féminin. Rien de neuf, certes, dans le constat que la poupée est un admirable instrument d'apprentissage symbolique des mécanismes de reproduction. Reproduction culturelle et sociale, celle des qualités et rôles sexués, bien sûr: rôle maternel quand la poupée fait de la petite fille une petite mère sous l'oeil de sa propre mère (pour qui la fillette peut être poupée...); rôle conjugal et érotique dans l'apprentissage du plaire par la parure. Mais au-delà de la fonction patente de la poupée dans nos textes, qui est de révéler un ou plusieurs aspects de la vie et de la « nature » des petites filles, on s'interrogera sur une autre fonction, latente: celle d'attirer sur le jeu en les focalisant certaines hantises et anxiétés propres à chaque auteur. C'est sous ces deux angles qu'elle sera principalement abordée ici à partir des pages inattendues, étonnantes, que lui ont consacrées Rousseau, Michelet et Beauvoir.

« Elle attend le moment d'être sa poupée elle-même » (Rousseau 707)

\section{Rousseau}

On sait que les idées de Rousseau sur les fillettes dans le cinquième livre de son traité-roman d'éducation Émile prennent le contre-pied presque exact des principes d'éducation dressés sur plusieurs centaines de pages pour l'enfant-modèle Émile. Liberté/servitude, être/paraître, action/passivité, utilité/frivolité - on n'en finirait pas d'aligner les oppositions. La plus profonde pourrait être celle-ci: ouverture / fermeture. L'enfant Émile est élevé pour être homme dans toute la largeur de ses possibles; la fillette est dirigée d'emblée vers un but contraignant: la domesticité (« les filles [...] doivent être génées de bonne heure. Ce malheur, si c'en est un pour elles, est inséparable de leur séxe » [Rousseau 709]). L'enfant Émile est protégé des influences néfastes et aliénantes de la société; la fillette semble n'avoir jamais vécu qu'en son sein ( « [...] la dépendance étant un état naturel aux femmes, les filles se sentent faites pour obéir » [Rousseau 710]). L'enfant Émile est naturellement indifférent à la sexualité, asexué lui-même; la fillette naît femme (" Le mâle n'est mâle qu'en certains instans, la femelle est femelle toute sa vie » [697]) et le devient donc d'autant mieux. C'est que l'éducation des filles est entièrement fonctionnelle: conçue en fonction de leur future « destination " domestique. Pour accomplir cette destination il faudra aux femmes avoir un ménage, et pour cela être mères, et pour cela se marier, et pour cela plaire aux hommes, et pour cela être attrayantes: « la femme est faite spécialement pour plaire à l'homme » (693). Voyons comment chez les fillettes selon Rousseau se concilient (heureuse convergence, signe d'une vérité) instinct naturel et impératif social autour du jeu de la poupée.

À propos des jeux Rousseau concède bien que « les enfants des deux séxes ont beaucoup d'amusemens communs » mais affirme aussitôt l'importance des goûts propres à chaque sexe. Les garçons aiment le mouvement et le bruit, 
donc tambours et sabots; les filles, « ce qui donne dans la vue et sert à l'ornement; des miroirs, des bijoux, des chiffons, surtout des poupées; la poupée est l'amusement spécial de ce séxe » (706). Nature et « destination » étant imbriquées l'une dans l'autre et mutuellement dépendantes, le passage de l'une (goût spontané) à l'autre (fonction sociale) se fait très rapidement et sans autre justification qu'un " très évidemment " péremptoire: "voilà très évidemment son goût déterminé sur sa destination. Le physique de l'art de plaire est dans la parure; c'est tout ce que des enfans peuvent cultiver de cet art » (706). C'est ici que survient un remarquable paragraphe:

Voyez une petite fille passer la journée autour de sa poupée, lui changer sans cesse d'ajustement, l'habiller, la deshabiller cent et cent fois, chercher continuellement de nouvelles combinaisons d'ornemens, bien ou mal assortis, il n'importe: les doigts manquent d'adresse, le goût n'est pas formé, mais déja le penchant se montre; dans cette éternelle occupation le tems coule sans qu'elle y songe, les heures passent, elle n'en sait rien, elle oublie les repas mêmes, elle a plus faim de parure que d'aliments: mais, direz-vous, elle pare sa poupée et non sa personne; sans doute; elle voit sa poupée et ne se voit pas, elle ne peut rien faire pour elle-même, elle n'est pas formée, elle n'a ni talent ni force, elle n'est rien encore; elle est toute dans sa poupée, elle y met toute sa coquéterie, elle ne l'y laissera pas toujours; elle attend le moment d'être sa poupée elle-même. (706-7)

Ce qui est décrit ici, c'est un état d'aliénation. ${ }^{\text {iv }}$ L'absorption entière des enfants dans les jeux d'imagination est facilement observable, mais ce n'est pas (simplement) de cela qu'il s'agit. La perte de conscience de soi, et du monde, et du temps acquiert une tonalité inquiétante, sinistre presque, en regard de 1'explication qui lui est donnée. L'état fébrile, aliéné de la fillette est le symptôme d'une impuissance à être ( elle ne peut rien faire pour elle-même », « elle n'est rien encore »), d'une tension désespérée vers le simulacre de son être à venir. Elle ne pare sa poupée que parce qu'elle ne peut parer sa personne, et ce, parce qu'elle n'est personne. Prise dans son jeu, une fillette pourrait se construire imaginairement dans une multiplicité de figures, grandie, enrichie par elles. Disparue dans son jeu, la fillette rousseauiste n'est qu'un immense vide, en qui la poupée provoque une sorte d'appel d'air. Mais ce n'est rien encore. La disparition de la fillette dans une image étrangère d'elle-même en prépare une autre plus longue: une aliénation autrement plus étendue l'attend à l'âge adulte, et c'est ce que la terrifiante formule finale fait brusquement surgir. La poupée n'est pas du tout un double de la fillette; c'est la femme que la fillette deviendra qui sera un simulacre de la poupée.

La femme selon Rousseau est maîtresse d'illusions, car par l'amour elle en vit. La fillette plongée dans son activité ludique est donc infiniment fidèle à sa nature féminine, est déjà dans son élément, celui de l'illusion (in-lusio): dans le 
jeu des apparences. Le malheur de Sophie ${ }^{v}$ fillette n'était pas tant l'absence d'être ou de substance que de paraître ou de forme(s) (« elle n'est pas formée »). La théâtralité du morceau de Rousseau est en parfaite consonance avec son propos.

La fillette de Rousseau est sérieuse, elle est active, elle est passionnée. Elle sait ce qui l'attend et elle attend de se faire. Mais de se faire, bien sûr, pour l'homme. Le lien entre parure et érotisme étant fort chez Rousseau, le jeu décrit ci-dessus est en fait extrêmement sexualisé. Pour qui connaît l'Émile et sa vibrante défense de l'enfance ( « Aimez l'enfance; favorisez ses jeux, ses plaisirs, son aimable instinct » [302]), le passage est d'autant plus troublant. « Les petites filles presqu'en naissant aiment la parure » (703, mes italiques). La fillette n'existe pas pour Rousseau; elle n'est qu'une femme en miniature, attitude qui dans la plus grande ironie est très précisément celle qu'il se targue d'avoir repoussée (« il faut considérer l'homme dans l'homme, et l'enfant dans l'enfant » [303]) et qu'il reproche à tous ceux qui ont écrit sur l'enfance: « La nature veut que les enfans soient enfans avant que d'être hommes [...]. L'enfance a des maniéres de voir, de penser, de sentir qui lui sont propres; rien n'est moins sensé que d'y vouloir substituer les nôtres " (319). Autre ironie: dans le zèle de l'auteur à faire de la coquetterie une nature afin que le plaire se transforme aisément en devoir, que devient la destination premièrement assignée aux femmes dans l'Émile, les soins de la famille? E. de Fontenay commente: "Mais on ne comprend plus: la poupée constitue-t-elle un amusement "spécial" en ce qu'elle préfigure la maternité, fin naturelle de la femme, ou bien en ce qu'elle signale l'amour-propre le plus perverti? » (1785). Une note de l'édition de la Pléiade sur le passage du jeu de la poupée indique erronément: « La petite fille se projette dans la poupée qu'elle pare, et anticipe sur la fonction maternelle » (Rousseau 1638), réinscrivant avec obstination ce qui pourtant ne figure pas du tout dans le passage annoté. Ceci montre bien le dérapage qui s'est produit à l'occasion de la poupée, dérapage attendu, de fait, puisqu'on sait que la Sophie de Rousseau est séductrice bien avant d'être mère, et bien plus.

Dans sa critique en règle du cinquième livre de l'Émile, Mary Wollstonecraft écrit:

As for Rousseau's remarks, which have since been echoed by several writers, that [girls] have naturally, that is from their birth, independent of education, a fondness for dolls [...] - they are so puerile as not to merit a serious refutation. That a girl [...] will imitate her mother or aunts, and amuse herself by adorning her lifeless doll, as they do in dressing her, poor innocent babe! is undoubtedly a most natural consequence. (42)

Osera-t-on ici parler de bon sens?

«Les enfants n'ont l'âme occupée/ Que de l'éternel souci/ Qu'on ne fầche point leur poupée » (Jean de La Fontaine, Fables IX, 6) 


\section{Michelet}

La femme selon Michelet est à la fois une grande enfant souffrante, et une mère toujours prête au sacrifice d'elle-même. Le premier livre de La Femme intitulée «De l'éducation » est consacré à la maternité. vi Dans les termes lyriques qu'on connaît à Michelet, les quatre premiers chapitres décrivent l'émerveillement de la jeune mère (enfant elle-même) face à son tout jeune enfant. Notons que le terme épicène d' " enfant » est repris systématiquement par le pronom « il » de sorte que ces premières pages sans le marquer présupposent toutes un enfant de sexe masculin (cf « mon fils » [Michelet 104]). Et de fait, au chapitre IV Michelet écrit: « Les filles, dont je parlerai tout à l'heure plus spécialement, ne sont pas plus élevées [...] qu'aux temps où l'auteur d'Émile a esquissé sa Sophie » (107). L'allusion à Rousseau est trompeuse. Le biais de Michelet est fort différent, puisqu'en la petite fille il ne voit pas la séductrice à venir mais la mère. Dans le chapitre III Michelet avait interpellé la jeune mère: « À quinze ans, s'il m'en souvient, sous prétexte d'essayer des modes, vous jouiez encore aux poupées. Même, quand vous étiez bien seule (convenez-en), il vous arrivait de les baiser, de les bercer. - La voici, la poupée vivante, qui ne demande qu'à jouer... Eh! jouez donc, pauvre petite! on ne vous regardera pas » (99). Ce n'est donc que par une sorte de dissimulation que la petite ou jeune fille feindra de s'intéresser à la parure quand c'est l'amour maternel qui s'éveille en elle par la poupée.

Le chapitre $\mathrm{V}$, qui nous retiendra ici, bref, lâchement composé, est intitulé « L'Amour à cinq ans. - La poupée ». Partant d'un certain nombre d'observations familières (une petite fille le voyant indisposé sur un canapé s'élance pour le border; la fillette en général aime à envelopper un objet quelconque de linges, lui marquer taille et tête, le bercer et l'embrasser), Michelet lance ainsi sa réflexion sur les poupées: « voici un jeu, mais sérieux, et bien plus sérieux qu'on ne pense. Quelle est cette nouvelle personne, cette enfant de notre enfant? Examinons tous les rôles que joue cette créature mystérieuse » (112). En vue des remarques sur la « poupée vivante », une valorisation de la préparation à la maternité s'impose. Certes, « vous croyez que c'est simplement une imitation de maternité. [...] Il y a de cela, mais ce n'est pas tout ». Et voici le reste: « Disons la chose comme elle est: c'est ici le premier amour » dont l'idéal sera une soeur caressante. Puis, « Autre point de vue, non moins vrai. C'est ici un premier essai d'indépendance, l'essai timide de l'individualité » (Michelet 112; en italiques dans le texte). Jusqu'ici rien sans doute d'extraordinaire. Ne négligeons point cependant la glose des notions $d^{\prime}$ ' indépendance » et d'《 individualité ». La poupée n'est pas innocente, elle peut être un instrument, minuscule et dérisoire, sans doute, mais instrument quand même, de subversion et de rébellion. La fillette, affirme Michelet, se sert de sa poupée comme d'une « petite, toute petite confidente » (sororale) pour exhaler secrètement ses plaintes. Sous la « forme toute gracieuse » du jeu de la poupée, voici que se découvre sourdement, « à [1']insu » de la fillette, « une velléité de poser à part, quelque peu d'opposition, de contradiction féminine. Elle commence son rôle de femme; toujours sous l'autorité, elle gémit un peu de sa mère, comme plus tard de son mari » qui, comme tous les hommes (« nous ») la fera bien pleurer, s'exclame Michelet en concluant son paragraphe (112). 
Si peu de jeu, si peu de joie. Le rapport de la fillette à sa poupée n'est pas tant ludique que lourdement psychologisé, et entièrement calqué sur les rapports adultes. Il constitue une sorte d'apprentissage de la tristesse solitaire de la femme mariée, et l'avertissement, peut-être, que toute velléité d'opposition à son sort devra demeurer vaine. Comme chez Rousseau, loin d'épanouir et d'enrichir l'enfant le jeu de la poupée d'emblée installe, enferme et contraint la fillette dans « son rôle de femme », lui-même restreint et contraignant. La vision implacable d'un futur gémissant est peu propice - c'est le moins qu'on puisse dire - à une analyse de la spécificité de l'enfance et de ses jeux, poupée ou autre. Constatons d'autre part que le texte, d'abord centré sur la maternité, a glissé vers l'expression d'une inquiétude, la ligue fillette/poupée étant calquée sur la ligue épouse/confidente. On revient à la maternité lorsqu'une conclusion semble se dessiner sous forme d'une admonition aux mères: "Il ne faut pas plaisanter, c'est une passion sérieuse. La mère doit s'y associer [...]. Loin de mépriser la poupée, elle insistera pour que l'enfant capricieuse lui soit toujours bonne mère, la tienne proprement habillée » etc. (Michelet 113). ${ }^{\text {vii }}$ Le thème de l'individualité réapparaît pourtant lorsque Michelet remarque: « Notez bien que plus la poupée de la petite fille est son oeuvre, plus elle est sa fabrication simple, élémentaire, mais aussi personnelle, plus elle y a mis son coeur, et plus il y a danger de la contrister » (113).

À ce moment une anecdote illustrative de cet aphorisme est entamée, histoire qui occupe presque toute la seconde moitié de ce bref chapitre V. De quoi s'agit-il? D'une fillette qui meurt d'amour pour une poupée, d'une fillette qui se dépérit réellement. Dans des pages expressément consacrées aux toutes petites filles de cinq ans et aux " gracieux » jeux de poupée, on trouve d'un coup maladie et cruauté, douleur et tragédie, provoquées précisément chez une toute jeune fillette et à propos d'une poupée. « Dans une campagne du Nord de la France ", entourée de frères moqueurs et surveillée par une mère austère, cette fillette se fait « une petite consolatrice » en chiffons, échange avec elles paroles, tendresses et larmes. Les garçons s'en aperçoivent, arrachent les bras de la poupée et la lancent dans un arbre. Elle y reste. L'enfant attristée pense à elle tout l'hiver. Au printemps, le jouet " s'était envol[é] au souffle du vent du Nord ». "Deux ans après " , la mère achète à sa fille une petite poupée d'Allemagne. Enchantée, l'enfant la pare, " ne pensait plus qu'à la faire belle et brillante ». Mais ses frères la font danser " à mort; ses bras s'arrachèrent " etc. « Nouveaux sujets de douleur - la petite fille en maigrit ». «Une demoiselle » lui offre enfin une ancienne poupée qui « paraissait vivante », mais quelqu'un pendant une brève maladie de la fillette,

je ne sais qui, peut-être par jalousie, brisa cruellement la poupée. Sa maîtresse, relevée du lit, la trouva décapitée. Cette troisième tragédie était trop, elle tomba dans un tel découragement qu'on ne la vit plus jamais rire, jamais jouer. Toujours trompée dans ses rêves, elle désespéra de la vie, qu'elle avait à peine effleurée, et rien ne put la sauver. Elle mourut, laissant un vrai deuil à tous ceux qui avaient vu cette douce, cette suave et innocente créature, qui n'avait guère été heureuse, et qui pourtant était déjà si tendre et le coeur plein d'amour (Michelet 115). 
Telle est la fin du chapitre.

N'en oublions pas le titre: « L'Amour à cinq ans ». Chez Michelet, quand à cinq ou sept ans on joue à la poupée, on finit comme une femme passionnée triplement trahie, amère, amaigrie et vieillie de chagrin; on se meurt, on est morte. ${ }^{\text {viii }}$

L'excès sidérant de cette « démonstration » renvoie à l'impossibilité déjà notée d'envisager la fillette sui generis, et illustre la compulsion de Michelet à projeter sur elle la femme qu'elle finit donc nécessairement par figurer. Cet excès signale alors que quelque chose se joue derrière l'exemple et qui ne peut pas être dit mais qui transparaît de façon à la fois énorme et oblique. Pourquoi la poupée est-elle ici si dangereuse qu'on ne cherche qu'à la détruire? Pourquoi la fillette estelle punie, et de mort? N'est-ce pas qu'elle manifestait un peu trop de cet esprit d'indépendance et de « rébellion » contre un ordre masculin violent (la ligue des garçons), et contre sa vocation domestique (d'où l'hostilité de la mère ${ }^{i x}$ ) en y échappant dans ses rêves, isolée près de sa poupée? Quelle sorte d'épouse aurait fait une telle enfant, demande implicitement Michelet? Cet amour fou, le mari en aurait-il bénéficié? Non pas, sans doute. Il n'appartient pas aux faibles de manifester tant d'intensité, tant de persévérance, et surtout de se perdre avec tant de passion dans un double (féminin) de soi. Michelet est comme Hugo sensible à la grâce lorsque l'enfant paraît et n'est pas incapable de communiquer le charme de la toute petite fille - le bref épisode où il se fait border par l'une d'elles en témoigne. Mais dans le souci de diriger son étude de la fillette en fonction de sa « destination » il ne voit en elle que l'adulte: elle est construite à des fins moralisantes. D'où le trouble d'une valorisation (la nature féminine est aimante) assortie d'une implicite condamnation: la « suave et innocente créature » est coupable envers sa propre nature par l'excès d'un amour mal orienté. Après tout, si « Élever une fille, c'est une oeuvre sublime et désintéressée », c'est bien parce que la fille « est destinée à un autre. Elle vivra pour les autres, non pour toi [la mère], et non pour elle » (Michelet 120; en italiques dans le texte).

« Je pris les deux petits volumes, je les flairai, je les palpai, les ouvris négligemment "à la bonne page" en les faisant craquer. En vain: je n'avais pas le sentiment de les posséder. J'essayai sans plus de succès de les traiter en poupées, de les bercer, de les embrasser, de les battre. Au bord des larmes, je finis par les poser sur les genoux de ma mère ") (Sartre 33).

\section{Beauvoir}

Qui d'autre que Beauvoir pouvait plus vigoureusement mettre en lumière tout ce qui dans l'expérience d'une fillette ordinaire est construction culturelle et conditionnement social? Dans le premier chapitre ("Formation ») du deuxième tome 
du Deuxième sexe, la preuve en serait que jusqu'à douze ans garçons et filles partagent à peu près tout, en particulier de ces traits conventionnellement attribués à l'un ou l'autre sexe. Ce sont les mêmes coquetteries, c'est la même robustesse etc. Si la spécificité sexuelle apparaît très tôt, " c'est que l'intervention d'autrui dans la vie de l'enfant est presque originelle et que dès ses premières années sa vocation lui est impérieusement insufflée " (Beauvoir 1976, 14). 11 n'est donc pas dit que la fillette ait envie d'un pénis en soi et pour soi; il est par contre certain qu'elle perçoit très tôt que cet organe procure des avantages dans le traitement que son possesseur reçoit des adultes. En outre, c'est un « organe qui se laisse voir et saisir ». « La petite fille cependant ne peut s'incarner dans aucune partie d'ellemême. En compensation on lui met entre les mains, afin qu'il remplisse auprès d'elle le rôle d'alter ego, un objet étranger: une poupée ".

Cette « figurine à face humaine » remplace donc

ce double, ce jouet naturel qu'est le pénis.

La grande différence c'est que, d'une part, la poupée représente le corps dans sa totalité et que, d'autre part, elle est une chose passive. Par là, la fillette sera encouragée à s'aliéner dans sa personne tout entière et à considérer celle-ci comme un donné inerte. Tandis que le garçon se recherche dans le pénis en tant que sujet autonome, la fille dorlote sa poupée et la pare comme elle rêve d'être parée et dorlotée; inversement elle se pense elle-même comme une merveilleuse poupée. (Beauvoir 1976, 27-28)

La fillette est par là confirmée dans cette tendance à se faire objet commune à tous les enfants (alors que le garçon doit la surmonter). Beauvoir reconnaît que la poupée est instrumentale mais n'est pas exclusivement déterminante dans l'opération (après tout, le jeune garçon peut avoir un ours etc.); « c'est dans la forme globale de leur vie que chaque facteur: pénis, poupée, prend son poids » (1976, 29). Il n'empêche. L'analyse de Rousseau est ici rejointe, évidemment non pas au plan normatif de ce dernier, mais comme diagnostic: la projection de soi dans la poupée symbolise, mais aussi prépare, mais aussi nourrit le dédoublement du sujet en objet qui caractérisera la femme-enfant, et la femme-poupée. La préoccupation de Beauvoir porte ici sur la passivité et l'absence d'initiative que semble entraîner chez la fillette le jeu de la poupée.

La poupée n'est pas seulement son double: c'est aussi son enfant, fonctions qui s'excluent d'autant moins que l'enfant véritable est aussi pour la mère un alter ego: à la fois quand elle gronde, punit, puis console sa poupée, elle se défend contre sa mère et elle se revêt elle-même de la dignité de mère: elle résume les deux éléments du couple; elle se confie à sa poupée, elle l'éduque, affirme sur elle son autorité souveraine, parfois même elle lui arrache les bras, la torture: c'est-à-dire qu'elle accomplit à travers elle l'expérience de l'affirmation subjective et de l'aliénation. Souvent la 
mère est associée à cette vie imaginaire: l'enfant autour de la poupée joue au père et à la mère avec sa mère. C'est un couple d'où l'homme est exclu. Là, non plus, il n'y a aucun "instinct maternel" inné et mystérieux. La fillette constate que le soin des enfants revient à la mère, on le lui enseigne; récits entendus, livres lus, toute sa petite expérience le confirme; on l'encourage à s'enchanter de ces richesses futures; on lui donne des poupées pour qu'elles prennent d'ores et déjà un aspect tangible. (1976, 32-33)

Après la sexualisation de la fillette par sa poupée (comme chez Rousseau), l'apprentissage de la maternité (comme chez Michelet) à l'aide de ce même jouet. On remarque combien Beauvoir insiste comme Michelet sur la multiplicité des rôles que peut assumer la fillette dans son jeu avec sa poupée, mais qu'elle met l'accent sur l'unité familiale (père-mère-enfant). L'influence de Freud est manifestement fort lourde dans la conception de la poupée comme substitut de pénis puis de bébé. ${ }^{x}$ Mais on note aussi et sans surprise que la description de Beauvoir vise à détruire tout le concept d' « instinct maternel » pour démontrer qu'il ne s'agit que de l'imitation d'une réalité observée tout au long de l'enfance: « le soin des enfants revient à la mère ».

Si Beauvoir ne contredit les descriptions ni de Rousseau ni de Michelet dans son analyse du rôle que peut jouer la poupée dans la vie d'une petite fille, c'est parce qu'elle semble les rejoindre sur le plan de l'observation empirique. Mais est-il besoin de dire à quel point sa pensée, et donc l'explication accordée à ces observations, diffèrent radicalement de celles de nos auteurs masculins? Rien de ce qui pour eux est nature de la fillette ne l'est pour Beauvoir; tout ce qui pour eux doit être la destination de la femme ne devrait pas l'être pour elle. Il convient pourtant de reconnaître le trouble que peuvent susciter tant de similitudes dans les descriptions des fillettes jouant à la poupée. Comme d'ailleurs dans l'ensemble du Deuxième sexe le problème est d'une part que Beauvoir passe infiniment plus de temps à décrire les symptômes de l'aliénation où sont enfermées les femmes qu'à élaborer des remèdes; et que de l'autre sa vision terrible, parfois atroce de la condition féminine imprègne tout naturellement les pages sur les fillettes prénubiles. ${ }^{\mathrm{xi}} \mathrm{Si}$ par exemple on traite la fillette " comme une poupée vivante » (Beauvoir 1976, 30), c'est qu'elle est destinée à devenir une « poupée de chair » (397) dans la dégradante obsession de son apparence qui empoisonnera sa vie d'adulte. Il va de soi que Beauvoir condamne ce destin, mais en adoptant presque exclusivement le mode du constat dans son chapitre « Formation » et en ne fournissant pas d'anti-modèle, elle y enferme sa fillette tout aussi sûrement que Rousseau ou Michelet. Le garçonnet des Mots traite ses livres comme des poupées, comme le montre l'épigraphe de cette section de notre étude; pourquoi n'est-il jamais concevable que des fillettes, dans leurs complexes jeux d'imagination et d'aventures, traitent leurs poupées comme des livres: incitations aux métamorphoses, moyens d'accès aux mondes enchantés? 


\section{Little women}

Chez nos auteurs, la poupée est si intéressante parce qu'elle est traitée comme un révélateur efficace. Elle annonce pour eux avec une remarquable acuité, comme une fulgurante évidence, ce qui fera l'existence d'une femme: parure et bébés, ou encore « bagatelles et préceptes » comme dit Montesquieu. La poupée est donc vicaire ou lieu-tenante de la femme dans la fillette, et du bébé dans la femme. Les discussions de la relation de la fillette avec sa poupée supposent toutes une vue des petites filles comme femmes en miniature, et ce précisément à cause d'une alternative implacable: séductrice ou mère, et épouse entre les deux, la fillette semble ne jamais pouvoir se rêver autrement. ${ }^{\text {xii }}$ Fatalité, et étroitesse de ce destin. L'enfance des filles est caractérisée par une extrême restriction de la relation au monde extérieur et par son corollaire: la domination de l'intériorité au double sens du terme: vie intérieure et intéricur domestique. Autre caractéristique: l'enfance féminine est vécue sous le régime de l'autre - altérité (la fillette n'est jamais elle-même), altération (elle doit s'embellir) et aliénation (elle vit dans l'autre).

«Plus jamais rire, jamais jouer » (Michelet): on ne peut qu'être surpris par la tonalité théâtrale, passionnelle, voire tragique des descriptions du jeu de la poupée chez les hommes. Chez Beauvoir par contre, le jeu est gris et terne et sue l'ennui des travaux ménagers auxquels il prépare. Trop de passion et trop d'action, ou pas assez. Dans un cas comme dans l'autre la construction de la féminité qui sous-tend ces descriptions et analyses est à la fois personnelle et idéologique, révélant anxiétés et préjugés. Chez Michelet comme chez Rousseau la fillette, ou plutôt la femme est irrationnelle, excessive, dangereuse par ses débordements et proche de la folie (soif effrénée de parures, désespoir mortel), potentiellement oublieuse de ses devoirs et donc toujours à surveiller et à contrôler. Elle est en même temps attachée au petit, au concret, au joli, au trivial et peu portée à la curiosité intellectuelle. Ce qui est nature pour ces deux écrivains ne l'est (naturellement) pas pour Beauvoir qui ne partage pas les obsessions de ses prédécesseurs, on s'en doute. Mais son anxiété à elle porte sur le destin féminin tel qu'elle prétend l'observer dans la société qui était la sienne: une vie conventionnelle et malheureuse décrite sur des centaines de pages dans Le Deuxième sexe. Les petites filles ne l'intéressent qu'en ce que peut se démontrer par elles combien leur expérience familiale et sociale les moule dès le plus jeune âge sans espoir de retour. C'est pourquoi chez elle aussi le portrait des fillettes est triste et lacunaire.

En effet, l'excès de la démonstration mène à d'importantes lacunes dans la représentation des petites filles au jeu. Esquissons-en quelques-unes. Ni Rousseau ni Michelet ni Beauvoir n'ont voulu voir dans le jeu rire et plaisir, fraîcheur et mystère. La croyance poétique et magique dans l'existence de la poupée, accompagnée du trouble et de l'enchantement dont a si bien parlé George Sand, peut s'étendre au-delà, ou rester en-deça des impératifs sociaux immédiats car elle appartient au domaine du mythe. Or ce domaine n'est jamais abordé dans nos textes parce que l'imaginaire de l'enfant y est à la fois alourdi et amputé. Il est de fait une quantité de fonctions remplies par le jeu des poupées qui ne sont jamais traitées parce qu'elles offriraient d'autres images des petites filles (et des petits 
garçons), plus ouvertes, plus libres, plus variées. L'étude ancienne mais encore précieuse de $\mathrm{S}$. Hall et $\mathrm{C}$. Ellis démontre assez que l'apprentissage de la maternité n'est qu'une fonction possible parmi d'autres. ${ }^{\text {xiii }}$

D'autre part, habillant ou dorlotant, dans les textes examinés la fillette semble n'être que tout amour et à tout moment. Seule Beauvoir note que la fillette peut très bien arracher elle-même les bras de sa poupée, n'attendant pas que ses frères le fassent (comme chez Michelet). Or le jeu de la poupée peut-être destructeur, peut être violent. Toute fillette lisant Les Malheurs de Sophie de la Comtesse de Ségur se reconnaitra sans doute davantage en Sophie ruinant sa poupée que dans des descriptions idylliques ou tragiques. La poupée de Sophie « peu à peu perdit ses charmes " sous l'action néfaste de sa propriétaire, qui lui ôte ses couleurs, la rend chauve, l'estropie, l'ampute de ses jambes. Point de passion éternelle à la Michelet: « Depuis tous ces malheurs, Sophie n'aimait plus sa poupée, qui était devenue affreuse » et qu'elle finit par fracasser sur des pierres; "Sophie ne pleura pas, mais elle invita ses amies à venir enterrer sa poupée " (Ségur 276). ${ }^{\text {iv }}$ C'est aussi chez cette dernière qu'on trouvera une autre dimension absente chez nos auteurs: la dimension collective et amicale du jeu de la poupée, occasion d'échanges, d'entraide, d'antagonismes aussi, comme dans tout jeu. $\mathrm{Ce}$ dernier n'aura dans ce cas rien à voir avec les exemples solitaires, tristes, maladifs, surveillés et punis qu'on trouve chez nos théoriciens.

Il est en outre parfaitement concevable qu'une fillette entretienne une relation avec sa poupée du type de celle (par exemple) qui unit « Calvin » à son tigre " Hobbes » dans la bande dessinée du même nom, c'est-à-dire que la poupée soit l'accompagnatrice, mieux, la partenaire d'aventures imaginaires infiniment drôles et variées, créatrices de leur propre univers et génératrices de leurs propres codes. Parallèlement, il est frappant que dans les textes considérés la poupée n'est jamais le double de la petite fille elle-même. Or Simone de Beauvoir a montré que la poupée peut très bien accompagner la vie de la petite fille comme petite fille seulement. "Dans mes jeux, je ne consentais à la maternité qu'à condition d'en nier les aspects nourriciers. Méprisant les autres enfants qui s'en amusent avec incohérence, nous avions, ma soeur et moi, une façon particulière de considérer nos poupées; elles savaient parler et raisonner, elles vivaient dans le même temps que nous au même rythme, vieillissant chaque jour de vingt-quatre heures: c'étaient nos doubles » (Beauvoir 1958, 77-78). Mais c'est précisément dans un texte autobiographique que cette intuition voit le jour, et non dans le texte analytique qu'est Le Deuxième sexe.

Enfin une perspective historique permet de détendre le lien implacable entre fillettes et poupées. C'est que les poupées sont loin d'avoir toujours été exclusivement " l'amusement spécial de ce sexe ». Il faut ici lire Philippe Ariès décrivant l'enfance de Louis XIII et les nombreuses mentions des poupées avec lesquelles ce dernier jouait, les peignant, les mariant etc. (56-62). Il conclut plus généralement qu'au tout début du XVIIe siècle au moins, " la poupée n'était pas réservée aux filles. Les garçons y jouaient aussi. A l'intérieur de la petite enfance la discrimination moderne entre les filles et les garçons était moins nette: les uns et les autres portaient alors [...] la même robe » (66-67). De son côté Mary Wollstonecraft (aînée de trois soeurs, gouvernante, maîtresse d'école) écrit: 
I have, probably, had an opportunity of observing more girls in their infancy than J.J. Rousseau - I can recollect my own feelings, and I have looked steadily around me; yet, so far from coinciding with him in opinion respecting the first dawn of the female character, I will venture to affirm, that a girl, whose spirits have not been damped by inactivity, or innocence tainted by false shame, will always be a romp, and the doll will never excite attention unless confinement allows her no alternative. Girls and boys, in short, would play harmlessly together, if the distinction of sex was not inculcated long before nature makes any difference. (43)

Enfin de leur côté deux psychologues de la fin du XIXe siècle observent: "That boys are naturally fond of and should play with dolls as well as girls there is abundant indication" (Hall and Ellis 49). ${ }^{\mathrm{xv}}$ Ni l'un ni l'autre des deux sexes n'est " spécialement » prédestiné à jouer à la poupée, mais les deux peuvent s'y livrer.

Freud prétendait avoir observé qu'à trente ans, un homme était en général encore souple et ouvert à l'expérience alors qu'une femme était effrayante de rigidité, comme si, dit-il l'apprentissage de la féminité avait bloqué celui de l'individu et épuisé la personne. ${ }^{\text {xvi }}$ Le jeu de la poupée, pour innocent (non nuisible) qu'il puisse paraître, s'il était véritablement joué par les fillettes comme le décrivent les trois auteurs considérés ici, serait capable de transformer ces propos sinistres de Freud en vérité absolue. Tous trois, incluant Beauvoir, se concentrent exclusivement sur l'aspect sexué de l'expérience de leurs sujets, et ce au détriment de l'enfance et de l'individu mêmes. Obsédés par la femme qu'ils voient dans la fillette (« Dès le berceau la femme est mère, folle de maternité », [Michelet], ils s'aveuglent à l'enfant. Les petites filles, finalement, ne jouent pas à la poupé chez Rousseau, Michelet et Beauvoir: y jouent seules les miniatures animées de leurs hantises.

\section{Notes}

${ }^{\text {i }}$ Pour un rapide panorama de l'histoire du jeu de la poupée depuis l'Antiquité jusqu'à l'Ancien Régime, voir Philippe Ariès (64-67), et de l'Antiquité au milieu du XXe siècle, M.-M. Rabecq-Maillard (30-42).

ii Le motif de la poupée comme anticipation de l'enfant à venir est omniprésent, on aura l'occasion de le constater ici même. Mais inversement, il ne faut pas oublier que c'est l'enfant qui peut être traité comme une poupée. Il existe au dixhuitième siècle, selon Elizabeth Badinter, une « représentation courante de l'enfant comme un jouet ou une machine. On sait qu'au XVIIIe siècle le petit enfant est désigné par le terme de "poupart" qui [...] signifiait [...] ce qu'on nommerait "poupée" » (67). Dans son Traité de l'éducation des enfants (1722), Crouzat se 
plaint des parents qui selon lui traitent leurs enfants comme eux-mêmes traitent leur poupée (Badinter 68).

iii Article « Poupée » de l'Encyclopédie.

iv Michelet et beaucoup d'autres décriront l'état d'absorption total de la mère par l'enfant, comme le médecin Gilibert: « Suivez ces mères qui nourrissent ellesmêmes leurs enfants ... Uniquement attentives à leurs enfants, elles passent les nuits sans dormir, leur repas est pris à la hâte... Toutes les heures du jour sont employées à laver, nettoyer, échauffer, amuser, nourrir, endormir l'objet de leur amours " (cité dans Badinter 187). Mais la petite fille de Rousseau ne materne pas sa poupée, et n'imite donc pas du tout cet « idéal » de dévouement maternel. v L'épouse idéale de l'élève Émile se nomme Sophie.

vi Sur l'idée que « toute femme est une malade », voir l'excellent ouvrage de Thérèse Moreau, p. 105-112 en particulier. Pour une brève étude de l'éducation de la fillette dans La Femme, voir ce même ouvrage p. 140-42.

vii Mais le jeu, même utile, a ses limites. Lorsque la fillette apprend la cuisine auprès de sa mère, Michelet précise: « je n'aime pas non plus, lorsqu'il s'agit d'une chose qui sera si grave, qu'on habitue cette enfant à s'en faire un jeu, à perdre le temps en petits gâchis pour le repas de sa poupée » (37).

viii Dans le texte sur Michelet de l'article « Femme » du Grand Dictionnaire Larousse (rédigé entre 1864 et 1876), Pierre Larousse commente discrètement: « Il s'apitoic sur les douleurs du premier âge [...]. C'est qu'aussi les enfants de M. Michelet sont très-avancés dans le sentiment; ils ont de bonne heure leur premier roman. Un chapitre intitulé: L'Amour à cinq ans en est la preuve » (cité dans Moreau 56.)

ix « Sa mère [...] la tenait toujours près d'elle au travail, pendant que les autres jouaient » (Michelet 113).

$\mathrm{x}$ "The feminine situation is [...] established [...] if the wish for a penis is replaced by one for a baby, if, that is, a baby takes the place of a penis in accordance with an ancient symbolic equivalence. It has not escaped us that the girl has wished for a baby earlier, in the undisturbed phallic phase: that, of course, was the meaning of her playing with dolls. But that play was not in fact an expression of her femininity; it served as an identification with her mother [...]. She was playing the part of the mother and the doll was herself. [...] Not until the emergence of the wish for a penis does the doll-baby become a baby from the girl's father, and thereafter the aim of the most powerful feminine wish" (Freud 592).

${ }^{x i}$ Ces pages sont proportionnellement très peu nombreuses - comme si de la toute jeune fillette il n'y avait que très peu à dire lorsqu'elle n'est pas considérée sous l'angle de la sexualité.

xii Il est peu de dire qu'on n'aurait que l'embarras du choix s'il fallait citer des auteurs qui ont présenté les femmes sous ces deux figures. Prenons (presque) au hasard Valéry: parmi les femmes, " les unes sont femelles par essence de l'animal humain », caractérisées par la tendresse et la fécondité. Les autres sont « des créatures sexuées que les fonctions de leur sexe ne doivent pas gêner pour la danse, pour l'esprit, pour accomplir leur devoir de jouets, de joyaux, et leurs destinées d'ornements et d'événements de la vie des hommes » (690). 
xiii Voir en particulier les pages 44-54 où sont développées différentes idées concernant l'influence du jeu de la poupée sur les enfants (selon leur âge).

xiv Cf aussi Sand: «J'ai aimé à casser les poupées » (554).

xv Ils continuent: "One boy in a family of girls, or boys who are only children, often play with dolls to seven or eight years of age. It is unfortunate that it is considered so predominantly a girl's play" (Hall and Ellis 49).

$\mathrm{xvi}$ "A man of about thirty strikes us as a youthful, somewhat unformed individual, whom we expect to make powerful use of the possibilities of development opened to him by analysis. A woman of the same age, however, often frightens us by her psychical rigidity and unchangeability [...] There are no paths open to further development; it is [...] as though, indeed, the difficult development to femininity had exhausted the possibilities of the person concerned" (Freud 599).

\section{Bibliographie}

Ariès, Philippe. L'Enfant et la vie familiale sous l'Ancien Régime. Paris: Plon, 1960. Badinter, Élizabeth. L'Amour en plus. Histoire de l'amour maternel (XVIIe-XXe siècle). Paris: Flammarion, 1980.

Baudelaire, Charles. « Les Misérables ». In Oeuvres complètes. Ed. Claude Pichois. Paris: Gallimard, Bibliothèque de la Pléiade, 1961.

Beauvoir, Simone de. Le Deuxième sexe, 2 vols. Paris: Gallimard, 1949, coll. « Folio », 1976. vol. II.

Mémoires d'une jeune fille rangée. Paris: Gallimard, 1958.

Fontenay, Élizabeth de. « Pour Émile et par Émile, Sophie ou l'invention du ménage ». Les Temps modernes 358 (1976): 1774-95.

Freud, Sigmund. "Femininity". In New Introductory Lectures on Psychoanalysis in The Complete Introductory Lectures on Psychoanalysis. Trad. James Strachey. New York: W.W. Norton \& Company, 1966.

Hall, G. Stanley et A. Caswell Ellis. A Study of Dolls. New York \& Chicago: E.L. Kellogg \& Co., 1897.

Hugo, Victor. Les Misérables. 2 vols. Paris: Gallimard, coll. « Folio », 1973. Vol. I.

Montaigne, Michel de. Oeuvres complètes. Ed. Albert Thibaudet et Maurice Rat. Paris: Gallimard, Bibliothèque de la Pléiade, 1962.

Michelet, Jules. La Femme. Paris: Flammarion, 1981.

Moreau, Thérèse. Le Sang de l'histoire: Michelet, l'histoire et l'idée de la ferme au XIXe siècle. Paris: Flammarion, 1982.

Rabecq-Maillard, M.-M. Histoire du jouet. Paris: Hachette, 1962.

Rousseau, Jean-Jacques. Émile, ou de l'éducation. In Oeuvres complètes. 5 vols. Ed. B. Gagnebin et M. Raymond. Paris: Gallimard, Bibliothèque de la Pléiade, 1969. Vol. IV.

Sand, George. Histoire de ma vie. In Oeuvres autobiographiques. Ed. Georges Lubin. Paris: Gallimard, Bibliothèque de la Pléiade, 1970. Vol. I.

Sartre, Jean-Paul. Les Mots. Paris: Gallimard, 1964. 